\title{
Análisis de la transferencia de calor de un sistema de refrigeración a partir de nanofluidos
}

\section{Heat transfer analysis of cooler system from nanofluids}

GARCIA-FLORES, José Luis $\dagger^{*}$, VALLE-HERNANDEZ, Julio, GALLARDO-VILLAREAL, José Manuel y ALONSO-ALFARO, Jorge Guillermo

Universidad Politécnica Metropolitana de Hidalgo, Maestría en Ingeniería Aeroespacial.

Universidad Autónoma del Estado de Hidalgo, Escuela Superior de Apan

ID $1^{\text {er }}$ Autor: José Luis, Garcia-Flores / ORC ID: 0000-0002-0339-5525, CVU CONACYT ID: 872522

ID $1^{\text {er }}$ Coautor: Julio, Valle-Hernandez / ORC ID: 0000-0001-8957-0066, Researcher ID Thomson: O-7339-2018, CVU CONACYT ID: 210743

ID $2^{\text {do }}$ Coautor: José Manuel, Gallardo-Villareal / ORC ID: 0000-0002-7578-7279, CVU CONACYT ID: 366394

ID $3^{\text {er }}$ Coautor: Jorge Guillermo, Alonso-Alfaro / ORC ID: 0000-0001-8073-6736, CVU CONACYT ID: 446175

DOI: 10.35429/JME.2019.12.3.16.24

Recibido 20 Julio, 2019; Aceptado 30 Diciembre, 2019

\section{Resumen}

En la última década una de las principales áreas de oportunidad en los sistemas de refrigeración, es la referente a aumentar la eficiencia de los mismos; para ello se ha estado innovando en materiales y fluidos de trabajo principalmente. En este trabajo se aborda el análisis de transferencia de calor en sistemas de refrigeración líquida agregando nanoparticulas. Dichos sistemas tienen diferentes aplicaciones industriales y en refrigeración de sistemas electrónicos. En el presente trabajo se propone una configuración del sistema de refrigeración a utilizar. El análisis consta del modelado matemático a partir del diseño de la geometría y la trayectoria de flujo en la tubería, además de una simulación en Dinámica de Fluidos Computacionales (CFD), del sistema. En los resultados se presentan las condiciones térmicas de operación en régimen laminar en 2D y se comparan con los resultados obtenidos con agua, sin nanoparticulas, estimando la ganancia que el sistema obtiene en la transferencia de calor al utilizar nanofluidos.

Nanofluidos, Refrigeración, Simulación CFD.

\begin{abstract}
In the last decade one of the main opportunity areas of the cooler systems is increase their efficiency; for this, it has been innovating in materials and working fluids mainly. In the last decade one of the main areas of opportunity in refrigeration systems is the reference to increase their efficiency. For this, it has been innovating in materials and fluids of work mainly. In this work, the analysis of the transfer of calories in liquid cooling systems is analyzed by adding nanoparticles. These systems have different industrial and refrigeration applications in electronic systems. In the present work a configuration of the refrigeration system to be used is proposed. The analysis consists of the mathematical mode from the design of the geometry and the trajectory of the flow in the pipeline, in addition to a simulation in Computational Fluid Dynamics (CFD) of the system. The conditions are presented in the results.
\end{abstract}

Nanofluids, Cooler, CFD simulation

Citación: GARCIA-FLORES, José Luis, VALLE-HERNANDEZ, Julio, GALLARDO-VILLAREAL, José Manuel y ALONSO-ALFARO, Jorge Guillermo. Análisis de la transferencia de calor de un sistema de refrigeración a partir de nanofluidos. Revista de Ingeniería Mecánica. 2019. 3-12: 16-24

\footnotetext{
* Correspondencia al Autor (Correo electrónico: joseluis_garcia01@live.com.mx)

$\dagger$ Investigador contribuyendo como primer Autor.
} 


\section{Introducción}

Los sistemas de refrigeración de alta eficiencia, se encuentran en desarrollo en diferentes áreas de la industria, tales como aeroespacial, electrónica, informática, comunicaciones, industria de alimentos, de generación de energía entre otras. El termino alta eficiencia hace referencia a la tasa de transferencia de calor que son capaces de remover, además del tiempo de vida y rangos de temperaturas en su operación. (Dr. Ashwini Kumar, 2017). El fluido refrigerante tiene un papel importante en el comportamiento de estos sistemas, es por ello que en el presente trabajo se propone utilizar nanofluidos como refrigerante.

Actualmente existe una gran cantidad de autores que reportan ganancia en la transferencia de calor al utilizar nanofluidos como refrigerante, sin embargo la configuración es de un tubo configurado linealmente (P.R.Mashaeia, 2016), en este caso se determina la ganancia en configuraciones diferentes.

Este tipo de sistemas son utilizados para refrigerar en su mayoría componentes electrónicos, los cuales a su vez están instalados en diferentes aparatos empleados en la industria de computadoras y aeroespacial principalmente. Aunque estos sistemas comparten la característica principal de ser de tamaño pequeño $\left(40 \mathrm{~cm}^{2}\right.$ promedio), no está descartado el uso de estos heat pipes, en aparatos de mayor tamaño y aprovechar la eficiencia del mismo en vehículos espaciales de mayor tamaño, instalaciones industriales entre otros. (Mameli1, 2018)

En el presente trabajo se desarrolla un modelo matemático para la transferencia de calor de los heat pipes, haciendo énfasis en la distribución de la temperatura en el interior del tubo. Con el seguimiento del comportamiento de esta variable, podemos visualizar la ganancia en la transferencia de calor del sistema propuesto con los materiales y nanoparticulas mencionados. Como resultados se muestran las gráficas de la distribución de la temperatura, así como del comportamiento de la velocidad del fluido a diferentes concentraciones.

\section{Descripción del sistema}

En este trabajo se propone el uso de un heat pipe, para refrigerar sistemas que operen en un rango de $18 \mathrm{~W}$ de calor, para los cuales se reportara la temperatura operativa y la distribución de la misma. Una de las principales aplicaciones de este estudio es en la industria aeroespacial, debido a las condiciones de operación., donde no existe espacio para un sumidero de calor, un ventilador o una bomba que apoye en la circulación del fluido de trabajo. (Shukla, 2018).

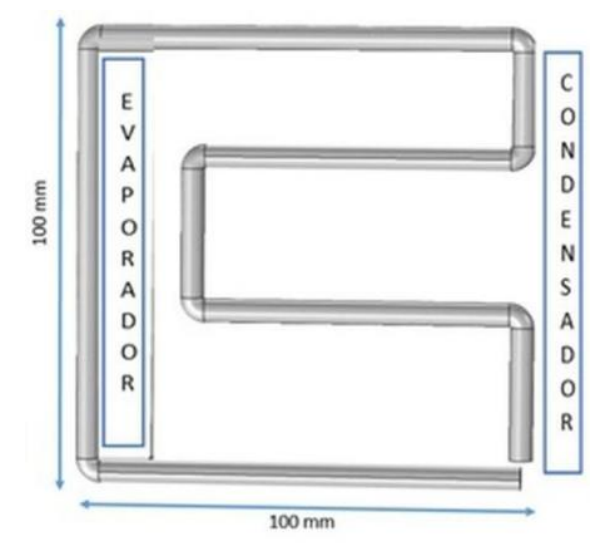

Figura 1 Configuración del sistema Fuente: Elaboración Propia

Este tipo de tubos favorecen la circulación natural del fluido, eliminando el uso de bombas y válvulas de expansión que los sistemas de refrigeración utilizan comúnmente. El tubo capilar analizado es de cobre calibre 0.28, (4.4 $\mathrm{mm}$ de diámetro interior), medidas comerciales utilizadas en equipos de aire acondicionado y refrigeradores, la medida es comercial debido a que esto facilitará la construcción de un prototipo experimental para validar los resultados.

\begin{tabular}{|l|r|}
\hline Material del tubo & \multicolumn{1}{c|}{ Cobre } \\
\hline Largo del tubo & $640 \mathrm{~mm}$ \\
\hline Largo evaporador & $103 \mathrm{~mm}$ \\
\hline Diámetro interior & $4.4 \mathrm{~mm}$ \\
\hline Diámetro exterior & $5.2 \mathrm{~mm}$ \\
\hline Largo condensador & $130 \mathrm{~mm}$ \\
\hline Radio de las curvas & $3 \mathrm{~mm}$ \\
\hline
\end{tabular}

Tabla 1 Geometría del sistema

Fuente: Elaboración Propia

\section{Metodología}

El estudio inicia con la propuesta de una geometría basada en referencias bibliográficas, (Stéphane Launay, 2018). 
Una vez que se propone la geometría se desarrolla el modelo matemático que rige este tipo de intercambiadores del calor, donde se identifican la variables principales que participan directamente en el desempeño del sistema, y sobre las cuales se realizaron gráficos para evaluar el desempeño del tubo. Una vez establecidas las ecuaciones que rigen el trabajo se analizan mediante simulaciones en CFD y se discuten los resultados.

\section{Modelos Matemáticos}

Existen diferentes modelos utilizados en este tipo de estudios, en este caso nos enfocaremos en los modelos establecidos (Faghri M. M., 1990) por los cuales muestran una diferencia importante en la temperatura de las secciones de alta y baja temperatura del heat pipe; las ecuaciones de estos modelos se enfocan en dos partes importantes del tubo, en forma radial por la naturaleza del estudio, donde el flujo másico y flujo de calor juegan un papel importante en este estudio.

\section{Sección de vapor}

En esta sección se tomaran valores típicos reportados para el número de Reynolds (210) y Mach (0.01), (Hitchcock, 1988) valores que describen a un flujo incompresible y laminar dentro del tubo en esta sección. Aunque se han reportado que no necesariamente debe ser así, pues también puede ser turbulento $(\operatorname{Re}<2000$, Mach<0.3) (P. D. Dunn and D. A. Reay, 1994)y mostrar un comportamiento diferente, pero cabe mencionar que en este régimen se ha estudiado el tubo en configuración lineal. Para nuestro caso de estudio la viscosidad será considerada constante de tal manera que en coordenadas cilíndricas (r-z) las ecuaciones quedan de la siguiente forma:

$\frac{1}{r} \frac{\partial}{\partial r}(r v)+\frac{\partial}{\partial z}(w)=0$

$\frac{1}{r} \frac{\partial}{\partial r}\left(\rho r v^{2}\right)+\frac{\partial}{\partial z}(\rho v w)=-\frac{\partial p}{\partial r}+$

$\mu\left[\frac{\partial}{\partial r}\left(\frac{1}{r} \frac{\partial}{\partial r}(r v)\right)+\frac{\partial^{2} v}{\partial z^{2}}\right]$

$\frac{1}{r} \frac{\partial}{\partial r}(\rho r v w)+\frac{\partial}{\partial z}\left(\rho w^{2}\right)=-\frac{\partial p}{\partial z}+$

$\mu\left[\frac{\partial}{\partial r}\left(\frac{1}{r} \frac{\partial}{\partial r}(r w)\right)+\frac{\partial^{2} w}{\partial z^{2}}\right]$

$\rho c_{p}\left(v \frac{\partial T}{\partial r}+w \frac{\partial T}{\partial z}\right)=k\left[\frac{1}{r} \frac{\partial}{\partial r}\left(r \frac{\partial T}{\partial r}\right)+\frac{\partial^{2} T}{\partial z^{2}}\right]$
Donde:

$\rho$ : Densidad.

$\mu$ : Viscosidad dinamica

p: Presión

T: Temperatura

Cp: Calor especfico

$\mathrm{k}$ : Conductividad termica

4.2 Sección de pared:

En la sección de pared, se considera conducción pura para este modelado, por lo que se describe con la siguiente ecuación:

$k_{w}\left[\frac{1}{r} \frac{\partial}{\partial r}\left(\frac{\partial T_{w}}{\partial r}\right)+\frac{\partial^{2} T_{w}}{\partial z^{2}}\right]=0$

\section{Condiciones de frontera}

Para este trabajo se requieren condiciones de frontera en ambos extremos del heat pipe, en la interfaz de vapor-líquido y el parte exterior de la pared del tubo y condiciones de no deslizamiento en el interior del tubo.

$v=w=0 ; \frac{\partial T}{\partial z}=0 ;$ cuando $z=0, L$

\section{Interfaz liquido vapor}

En la interfaz de líquido-vapor, se consideran condiciones de saturación durante la operación del heat pipe, por lo cual la temperatura en este punto se puede modelar con la ecuación de Clausius- Clapeyron (Faghri A. , 1995)

$$
T=\frac{1}{\frac{1}{T_{0}}-\left(\frac{R}{h_{f g}}\right) \ln \left(\frac{p_{v}}{p_{0}}\right)}
$$

Donde:

\section{$R$ : Constante del gas}

$h_{f g}$ : Calor latente del fluido de trabajo.:

$p_{0}$ : Presion de saturación.

$T_{0}$ :Temperatura de saturación. :

Las condiciones aplicadas para la velocidad se basan en la tasa de cambio de evaporación y condensación del fluido de trabajo, la velocidad axial y longitudinal en la interfaz liquido-vapor está dada por:

$v_{i}=\frac{\dot{m}}{\rho}$

Donde:

$\dot{m}=$ Flujo masico en la interfaz vapor - liquido. 
Considerando que los efectos del fluido en la zona caliente la velocidad se considera negativa, mientras que en la zona fría se considera positiva, para el balance de masa. Finalmente las consideramos el flujo de calor en la pared del tubo, utilizando:

$k_{w}=\frac{\partial T_{w}}{\partial r}= \pm \frac{Q}{A}$

Una distribución de calor en el tubo, se considera positivo en la sección de vapor, cuerpo en la sección adiabática y negativo en la zona de condensación.

\section{Propiedades de los nanofluidos empleados}

Desde que se dieron a conocer los primeros estudios y se originó el término de nanofluido (S. U. S. Choi, 1995) la mayoría de las investigaciones de este tema se han enfocado en el estudio de sus aplicaciones y ya que estas son fuertemente dependientes de la temperatura y la concentración volumétrica de las nanopartículas suspendidas, Tales propiedades muestran grandes diferencias con las de fluidos en magnitud y también en su comportamiento (Philip, 2008).

Experimentalmente, se ha encontrado que para nanofluidos la conductividad térmica llega a ser el doble que la del fluido base puro, esto solo con incluir una pequeña cantidad de nanopartículas en el líquido portador. Una parte importante en el estudio de los nanofluidos es la concentración de nanoparticulas suspendidas en el fluido base, en este trabajo se tomaran concentraciones pequeñas, $0,05,0,075$ y $0.1 \mathrm{vol}$. Emplearan relaciones semiteoricas (Maheswaran, 2018)para ingresar al software CFD, las propiedades necesarias para simular el comportamiento del nanofluido en el sistema.

\section{Viscosidad}

Debido a la presencia de partículas, la viscosidad de los nanofluidos es superior a la de los fluidos base, para la determinación de la viscosidad de suspensiones coloidales se han desarrollado algunas expresiones teóricas, las cuales presentan resultados aceptables en relación a mediciones experimentales. (Bird, 2007).
Así, Einstein desarrollo una expresión teórica para la evaluación de la viscosidad efectiva, $\mu_{e f f}$ de una suspensión diluida de partículas esféricas. Idealmente las nanoparticulas con tamaño promedio entre $10 \mathrm{y}$ $50 \mathrm{~nm}$ son de forma esférica, por lo cual para este trabajo de investigación la siguiente expresión es válida.

$\mu_{n f}=\frac{\mu_{f b}}{(1-\phi)^{2.5}}$

Donde:

$\mu_{n f}$ :Viscosidad nanofluido.

$\mu_{f b}$ :Viscosidad fluido base.

$\phi$ : Concenytración (vol).

Cabe mencionar que la ecuación anterior es válida para concentraciones volumétricas menores al 5\%, por lo cual es totalmente aplicable en este trabajo.

\section{Densidad}

La densidad es una magnitud escalar referida a la cantidad de masa de una sustancia contenida en un determinado volumen. En los nanofluidos mediante la teoría de mezclas ideales (Smith, 2005) se puede deducir fácilmente expresión para su cálculo, la expresión es:

$\rho_{n f}=(1-\phi) \rho_{f b}+\phi \rho_{n p}$

Donde:

$\rho_{n f}, \rho_{f b}, \rho_{n p}:$ Densidad nanofluido, fluido base y nanoparticula

$\phi$ : Concentración volumetrica

\section{Calor específico}

El calor específico es una magnitud física que se define como la cantidad de calor que hay que suministrar a la unidad de masa de una sustancia o sistema termodinámico para elevar su temperatura en una unidad (kelvin). En los nanofluidos es posible calcular el calor específico mediante la teoría de mezclas (Wylen, 2008), es decir, mediante un balance de energía en estado estacionario y suponiendo el calor específico constante con la temperatura, se llega a una expresión de la forma, en donde las variables de la misma ya han sido descritas.

$\rho C p_{n f}=(1-\phi)(\rho C p)_{f b}+\phi(\rho C p)_{n p}$ 


\section{Conductividad térmica}

Es la capacidad de una sustancia de transferir la energía cinética de sus moléculas a otras moléculas adyacentes o a sustancias con las que está en contacto. (Incropera, 1999). Es uno de los parámetros más importantes en la transferencia de calor, y la mayor parte de los trabajos experimentales de nanofluidos procuran estudiar esta propiedad como función de la temperatura y la concentración volumétrica de las nanopartículas en suspensión.

Maxwell desarrolló un modelo para determinar la conductividad eléctrica o térmica de medios heterogéneos con fracción volumétrica mínima de partículas (Maxwell, 1891). Aunque ha sido aplicado con éxito en compuestos sólidos, es aplicable en menor medida en suspensiones líquidas homogéneas, diluidas, y de partículas esféricas.

$K_{n f}=\frac{2 K_{n p}+K_{f b}+\phi\left(K_{n p}-K_{f b}\right)}{2 K_{n p}+K_{f b}-2 \phi\left(K_{n p}-K_{f b}\right)}$

Los subíndices de la expresión anterior ya han sido descritos anteriormente.

\section{Modelo CFD}

En la simulación de este sistema se utilizan condiciones de frontera de Von Neumann, es decir conocemos el flujo de calor y temperatura en la sección de evaporador, $18 \mathrm{~W}$ y $97.5^{\circ} \mathrm{C}$, además de las propiedades del fluido calculadas con las expresiones anteriores. Esta simulación se hizo en estado transitorio, en trabajos futuros se pretende analizar el estado transitorio y con otras geometrías y concentraciones de nanoparticulas. El régimen del fluido dentro del tubo será laminar, en este trabajo Las nanoparticulas que se seleccionaron para este trabajo son de plata $(\mathrm{Ag})$ y cobre $(\mathrm{Cu})$, de tamaño promedio de $20 \mathrm{~nm}$, para la cales existen propiedades teóricas definidas y se muestran a continuación:

\begin{tabular}{|l|rr|r|r|}
\hline $\begin{array}{c}\text { Propieda } \\
\mathbf{d}\end{array}$ & $\begin{array}{c}\text { Densida } \\
\mathbf{d} \\
{\left[\mathbf{K g} / \mathbf{m}^{\mathbf{3}}\right]}\end{array}$ & $\begin{array}{c}\text { Cp } \\
{[\mathbf{J} / \mathbf{g K}}\end{array}$ & $\begin{array}{c}\text { Viscosida } \\
\mathbf{d} \\
{[\mathbf{m P a s}]}\end{array}$ & $\begin{array}{c}\text { Conductivida } \\
\mathbf{d} \\
{[\mathbf{W} / \mathbf{m K}]}\end{array}$ \\
\hline Plata & 10.5 & 0.49 & 2 & 12.3 \\
\hline Cobre & 8.96 & 0.24 & 2.57 & 48.3 \\
\hline
\end{tabular}

Tabla 2 Propiedades nanoparticulas Fuente: American elements
En la siguiente tabla se muestran las propiedades de los nanofluidos empleados en este trabajo, las cuales se obtuvieron utilizando las propiedades anteriores.

\begin{tabular}{|l|r|r|r|}
\hline \multicolumn{1}{|c|}{ Propiedad / $\boldsymbol{\phi}$} & \multicolumn{1}{c}{$\mathbf{0 . 0 5}$} & \multicolumn{1}{c|}{$\mathbf{0 . 7 5}$} & \multicolumn{1}{c|}{$\mathbf{0 . 1}$} \\
\hline Viscosidad.[Pa*s] & 2.273 & 2.43 & 2.60 \\
\hline Densidad.[Kg/m $\left.{ }^{3}\right]$ & 1472.15 & 1709.72 & 1947.3 \\
\hline Calor especifico[J/Kg*K] & 2.86 & 2.48 & 2.19 \\
\hline $\begin{array}{l}\text { Conductividad Térmica. } \\
{[\mathrm{W} / \mathrm{mK}]}\end{array}$ & 14.8 & 14.97 & 15.48 \\
\hline
\end{tabular}

Tabla 3 Propiedades nanofluido de Plata

\begin{tabular}{|l|r|r|r|}
\hline \multicolumn{1}{|c|}{ Propiedad / $\boldsymbol{\phi}$} & \multicolumn{1}{c}{$\mathbf{0 . 0 5}$} & \multicolumn{1}{c|}{$\mathbf{0 . 7 5}$} & \multicolumn{1}{c|}{$\mathbf{0 . 1}$} \\
\hline Viscosidad.[Pa*s] & 2.29 & 2.41 & 2.60 \\
\hline Densidad.[Kg/m $\left.{ }^{3}\right]$ & 1392.45 & 1590.17 & 1789.9 \\
\hline Calor especifico[J/Kg*K] & 2.86 & 2.67 & 2.38 \\
\hline $\begin{array}{l}\text { Conductividad Térmica. } \\
{[\mathrm{W} / \mathrm{mK}]}\end{array}$ & 57.22 & 57.90 & 58.59 \\
\hline
\end{tabular}

Tabla 4 Propiedades nanofluido de Cobre

Se aplican las condiciones de frontera mencionadas anteriormente $y$ en primera instancia se corre el modelo utilizando solo agua, para tener un parámetro de comparación del comportamiento del nanofluido a través del sistema. Luego se introducen las propiedades del nanofluido de ambas partículas metálicas, en el software se da como fluido de trabajo el agua destilada, pero se introducen manualmente las propiedades de cada nanofluido en cada concentración (Tabla 3), de igual manera se hacen las corridas con las condiciones de frontera mencionadas y se grafica la distribución de temperatura en el tubo.

\section{Resultados y discusión}

En la figura 2, se observa la distribución de temperatura, donde se aprecia la mayor temperatura en la zona de vapor, donde estará colocado el componente del cual se quiere retirar calor y en la parte de condensación la temperatura es más baja. Esta es una de las principales ventajas de estos sistemas, ya que entre más se enfrié el fluido en el interior del tubo, existirá una mayor diferencia de temperaturas, lo cual conlleva a que se pueda extraer más calor. Otro de los parámetros más importantes es la velocidad dentro del tubo es la velocidad del fluido en el interior del mismo, es de vital importancia conocer el campo de velocidades, el cual se muestra en la figura 3. 


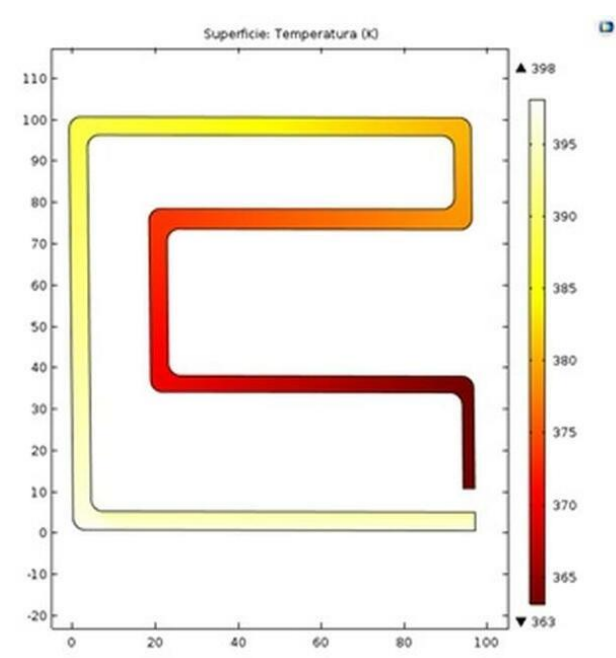

Figura 2 Distribución de temperatura del sistema, utilizando agua

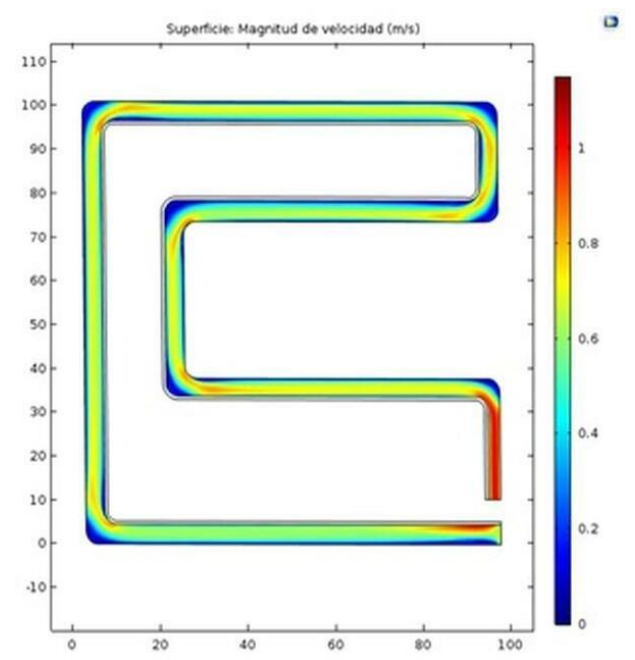

Figura 3 Campo de velocidades del sistema, utilizando agua

Posteriormente se repitió la simulación, pero ahora con las nanoparticulas dispersas, como ya se mencionó antes en concentraciones pequeñas, menos del 5\%, las simulaciones arrojaron los siguientes resultados.

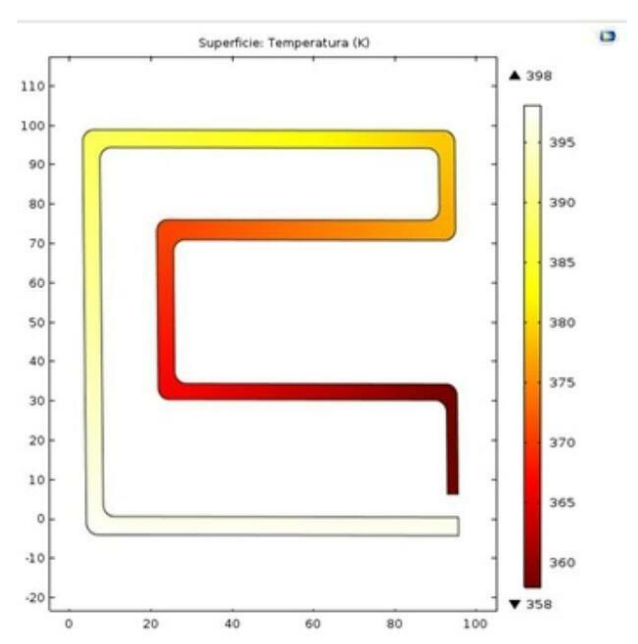

Figura 4 Distribución de temperatura del sistema, utilizando nanofluido de plata a $0.1 \%$ de concentración volumétrica

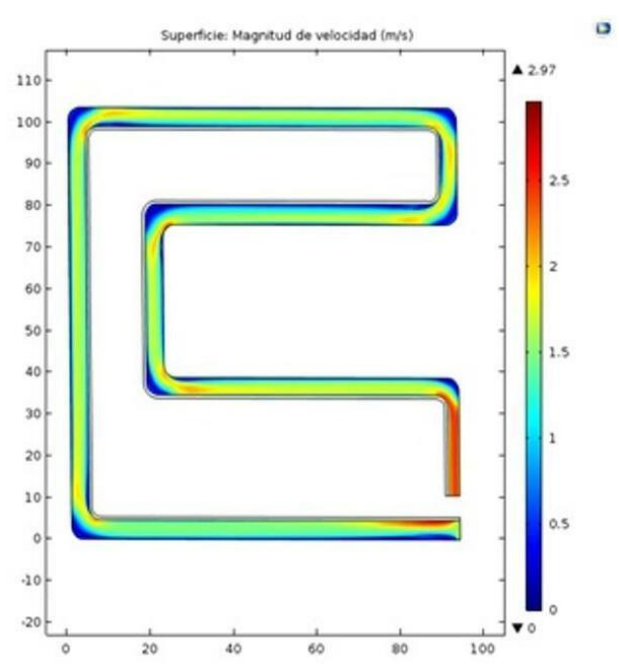

Figura 5: Distribución de velocidad en el interior del tubo, utilizando nanofluido de plata a $0-1 \%$ de concentración volumétrica

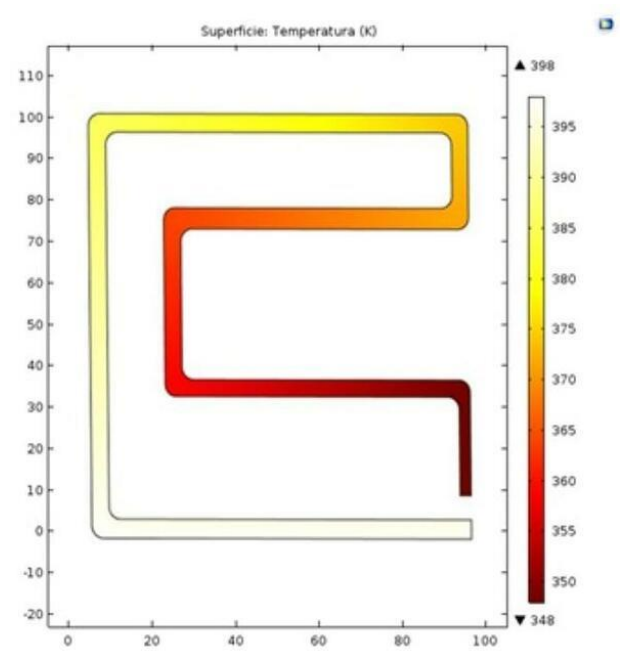

Figura 6 Distribución de temperatura del sistema, utilizando nanofluido de cobre a $0.1 \%$ de concentración volumétrica

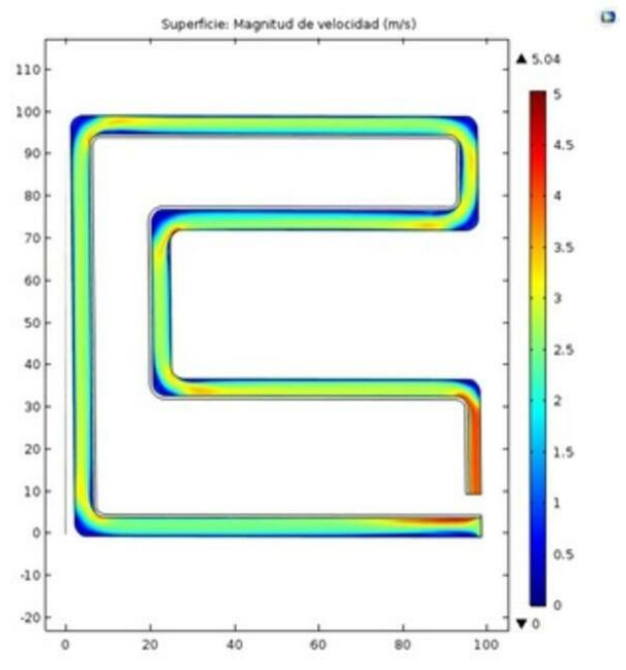

Figura 7 Distribución de velocidad en el interior del tubo, utilizando nanofluido de cobre a $0.1 \%$ de concentración volumétrica

GARCIA-FLORES, José Luis, VALLE-HERNANDEZ, Julio, GALLARDO-VILLAREAL, José Manuel y ALONSO-ALFARO, Jorge Guillermo. Análisis de la transferencia de calor de un sistema de refrigeración a partir de nanofluidos. Revista de Ingeniería Mecánica. 2019 
Los resultados obtenidos de las primeras corridas en el software, las cuales fueron hechas empleando agua como fluido dentro del tubo, arrojaron un comportamiento favorable en la distribución de temperatura, lo cual indica que el sistema es viable para extraer calor, como se observa en la figura 2 .

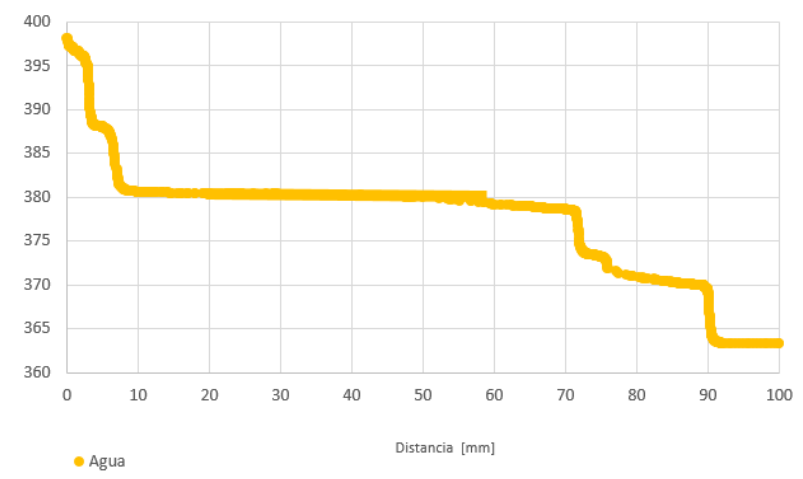

Gráfica 1 Distribución de temperatura utilizando agua como fluido refrigerante

En la figura anterior se observa que la trayectoria propuesta en el sistema es adecuada para la aplicación de refrigerar, la diferencia de temperatura entre la zona de vapor y de condensación es de $35^{\circ} \mathrm{C}$. Este resultado nos sirve de parámetro en las simulaciones ahora con nanofluido. La trayectoria escalonada d la temperatura se debe a la forma del tubo.

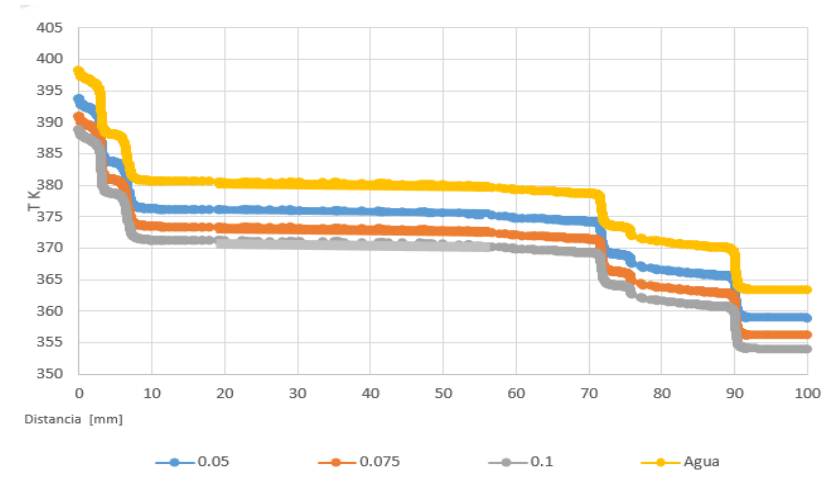

Gráfica 2 Distribución de temperatura utilizando nanofluido de plata en diferentes concentraciones

La figura 3 muestra el comportamiento de la temperatura en el interior del tubo, comparando la trayectoria con la gráfica anterior donde se utilizó agua como fluido de trabajo, se puede apreciar el descenso de la misma cuando se agregan nanoparticulas de plata al agua.

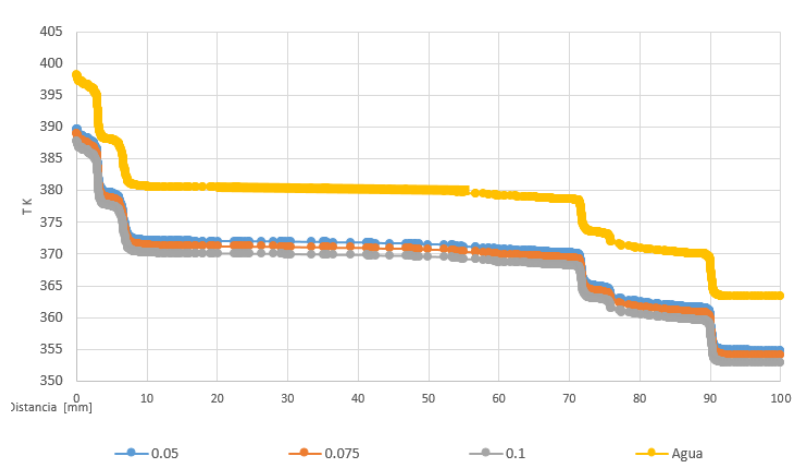

Gráfica 3 Distribución de temperatura utilizando nanofluido de cobre en diferentes concentraciones

\section{Conclusiones}

1.- En este trabajo se concluye que utilizar nanofluidos como fluido de trabajo en un tubo de calor de este tipo mejora la transferencia de calor, ya que existe una diferencia de temperatura entre el sistema con agua y con los dos diferentes nanofluidos propuestos en este trabajo, en concentraciones de $0.05,0.075$ y 0.1 vol. Por lo cual este trabajo se pude continuar en simulaciones en $3 \mathrm{~d}$ más detalladas y validar estos resultados con un prototipo experimental según la aplicación específica que pueda tener este sistema.

2.- Se propone esta geometría la cual arrojo resultados buenos en la transferencia de temperatura, además de que el diámetro del tubo existe de manera comercial, lo que hace factible la construcción de un prototipo experimental. $\mathrm{La}$ temperatura máxima del sistema es $125^{\circ} \mathrm{C}(398$ $\mathrm{K})$ mientras que la temperatura mínima en estas condiciones es $90{ }^{\circ} \mathrm{C}(363 \mathrm{~K})$, este último valor se compara con la temperatura mínima del sistema con los nanofluidos de cobre y plata.

a) Plata

\begin{tabular}{r|r|}
$\begin{array}{c}\text { Concentración } \\
\phi\end{array}$ & $\begin{array}{c}\text { T mínima } \\
{\left[{ }^{\circ} \mathbf{C}\right]}\end{array}$ \\
\hline 0.05 & 85.6 \\
\hline 0.075 & 82.9 \\
\hline 0.1 & 80.75 \\
\hline
\end{tabular}

b) Cobre

\begin{tabular}{|r|r|}
\hline $\begin{array}{c}\text { Concentración } \\
\phi\end{array}$ & $\begin{array}{c}\text { T mínima } \\
{\left[{ }^{\circ} \mathbf{C}\right]}\end{array}$ \\
\hline 0.05 & 81.8 \\
\hline 0.075 & 78.2 \\
\hline 0.1 & 75.75 \\
\hline
\end{tabular}

Tabla 5 Temperatura mínima del sistema con cada nanofluido 
La suspensión de nanoparticulas de plata presenta una reducción de temperatura mínima respecto del agua de $4.4{ }^{\circ} \mathrm{C}$ en la concentración más pequeña $(0.075 \mathrm{vol}$.), mientras que en la concentración mayor ( 0.1 vol.) la diferencia es de $9.25^{\circ} \mathrm{C}$. En las nanoparticulas de cobre la reducción de temperatura de $9.85{ }^{\circ} \mathrm{C}$ más que cuando se emplea agua en la concentración más pequeña y $14.25{ }^{\circ} \mathrm{C}$ en la concentración mayor, por lo que se recomienda utilizar las nanoparticulas de cobre en el caso del prototipo experimental.

3.- A partir de los resultados obtenidos se recomienda realizar un prototipo experimental que valide estos resultados, adaptarlo a las condiciones operativas de la aplicación, probando nuevas geometrías y trayectorias del tubo.

\section{Referencias}

Bird, B. S. (200@7). Fenómenos de Transporte. Mexico: : Limusa Wiley.

Das, S. K. (2008). Nanofluids: Science and technology. New Jersey: John Wiley \& Sons.

Dr. Ashwini Kumar, R. K. (2017). A Review of Electro Thermal Cooling Systems with Heat. International Journal of Engineering Research And Management (IJERM), 49-58.

Faghri, A. (1995). Heat Pipe Science and technology. Taylor \& Francis, 268-281.

Faghri, M. M. (1990). An analysis of the vapor flow and the heat conduction through the liquidwick and pipe wall in a heat pipe with single or multiple heat source. International Journal of heat mass transfer.

Hitchcock, W. J. (1988). Transient compressible heat-pipe vapor dynamics. ASME National Heat transfer conference.

Hussein, A. M. (2015). The Effect of Nanofluid Volume Concentration on Heat Transfer and Friction Factor inside a Horizontal Tube. Journal of Nanomaterials.

Incropera, F. P. (1999). Fundamentos de Transferencia de Calor (4 Ed ed. Mexico: Prentice Hall.
Kakac, S. \&. (2009). Review of convective heat transfer enhancement with nanofluids. International Journal of Heat and Mass Transfer, 311.

Maheswaran, R. (2018). Estimation of ThermoPhysical Properties of Nanofluids using Theoretical Correlations. International Journal of Applied Engineering Research, 13.

Mameli1, M. (2018). Large Diameter Pulsating Heat Pipe for Future Experiments on the International. School of Computing, Engineering and Mathematics, University of Brighton, 10.

Maxwell, J. C. (1891). Electricity and Magnetism (Vol. unabridged $3 \mathrm{rd}$ ed). . UK: Oxford.

Mishkinis, D. ( 2018). Loop Heat Pipes: Fundamentals and Applications. International Electronics Cooling Technology, 68.

P. D. Dunn and D. A. Reay, «. 4. (1994). Heat Pipe. New York: Pergamon, pp. 45-60.

P.R.Mashaeia, *. M. (2016). Analytical study of multiple evaporator heat pipe with nanofluid; Asmart material for satellite equipment cooling application. Aerospace Science and Technology, 112-121.

Philip, J. S. (2008). Evidence for enhanced thermal conduction through percolating structures in nanofluids. Nanotechnology, 19.

S. U. S. Choi, \&. J. (1995). Enhancing thermal conductivity of fluids with nanoparticles. Materials Science, 99-105.

Shukla, K. N. (2018). Heat Pipe for Aerospace Applications-An. Journal of Electronics Cooling and Thermal Control, 1-14.

Sidik, N. A. (2017). Recent advancement of nanofluids in engine cooling system. Renewable and Sustainable Energy Reviews, 137-144.

Smith, J. M. (2005). Introduction to chemical engineering thermodynamics. . New York: McGraw-Hill.

Stéphane Launay, *. V. (2018). Analytical Model for Characterization of Loop Heat Pipes. JOURNAL OF THERMOPHYSICS AND HEAT TRANSFER. 
Wylen, G. V. (2008). Fundamentals of Thermodynamics. United Sates: Wiley.

Xie, W. Y. (2014). A Review on Nanofluids: Preparation, StabilityMechanisms,. Journal of Nanomaterials, 17.

Yimin Xuan *, Q. L. (2018). Heat transfer enhancement of nanofuids. International Journal of Heat and Fluid Flow, 58-64. 